\title{
Synthesis and Gas Permeability of Aromatic Polyamide and Polyimide Having Oligodimethylsiloxane in Main-Chain or in Side-Chain
}

\author{
Yusuke Kawakami, ${ }^{\dagger}$ Shan-Pu Yu, ${ }^{*}$ and Toshihiro AbE* \\ Graduate School of Materials Science, \\ Japan Advanced Institute of Science and Technology, Hokuriku Asahidai 15, \\ Tatsunokuchi, Ishikawa 923-12, Japan \\ * Department of Applied Chemistry, School of Engineering, Nagoya University, \\ Chikusa, Nagoya 464, Japan
}

(Received April 23, 1992)

\begin{abstract}
Aromatic polyamides and polyimide having oligodimethylsiloxane of defined length ( $1-4 \mathrm{Si}$ atoms) in main-chain or in side-chain were synthesized by polycondensation of bis(4-aminophenyl)-functionalized oligodimethylsiloxanes with acid dichloride or pyromellitic dianhydride. Glass transition temperature and gas permeability of the polymers were markedly influenced by the introduction of oligodimethylsiloxane components into the polymer. The effects was more notable in polymers having oligodimethylsiloxane as side-chains.
\end{abstract}

KEY WORDS Oligodimethylsiloxane / Polyamide / Polyimide / Glass Transition Temperature / Gas Permeation/

During the last decade, remarkable progress has been made in the technology of gas separation by selective permeation through polymer membranes. However, only few significant studies on the structure-permeability relationships have been published until now, and insufficient understanding of the relationships between the chemical structure of polymers and their gas permeability makes the progress slow, hence new materials for membranes are developed only on a trial-anderror basis. For further progress, creation of new polymers which exhibit both a high selectivity for specific gases and a high intrinsic permeability based on molecular design of the polymer is essential. ${ }^{1}$

We reported that introduction of oligodimethylsiloxane as substituents into polymer structure is an excellent method to develop selectively oxygen permeable membrane mate- rials of well-balanced property between permeability, selectivity in permeation and mechanical strength of the membrane. In order to clarify the role of siloxane linkage in permeation, and to establish the relationships between structure and permeability, glass transition temperature, several polymers having oligodimethylsiloxane side-chains have been synthesized, and their properties were estimated. $^{2-7}$

It is of interest to study the effect of siloxane components on hydrogen bonding and on the mechanical strength of the polycondensation polymers, since polydimethylsiloxane is considered to destroy, or at least weaken hydrogen bonding or polar interaction in these polymers. Although, there are already reports in which polydimethylsiloxane is introduced into aromatic polycarbonate, polyamide as a component of a block copolymer, or as branches of

\footnotetext{
$\dagger$ To whom correspondence should be addressed.
} 


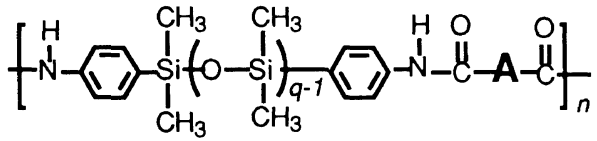

m-PIAq or m-PTAq<smiles>CN(CCC(=O)Nc1ccc([Si](C)(O[Si](C)(C)C)c2ccc(N(C)C(C)(C)C)cc2)cc1)C(C)(C)C</smiles>

s-PIAq<smiles>CN(C)c1ccc(C(C)(C)C)cc1</smiles>

S-PIAMq

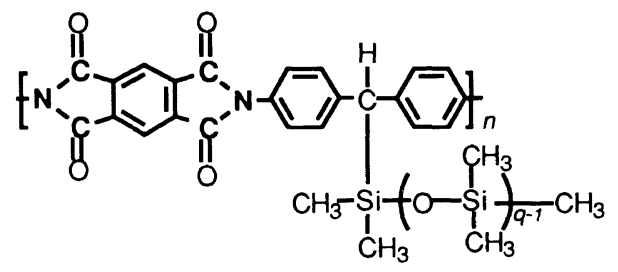

PIMq

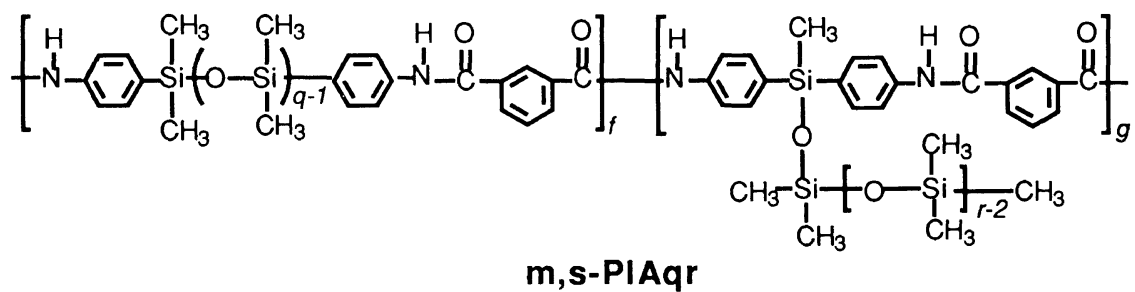

m-, main-chain type; s-, side-chain type; m,s-, polymer from $1: 1$ mixture of $\alpha, \omega$-bis(4aminophenyl)oligodimethylsiloxane and 1,1-bis(4-aminophenyl)oligodimethylsiloxane as amine components

$$
\text { A: (PT series) or (PI series) }
$$

Figure 1. Structure of polymers.

a graft copolymer with polyether mainchain, ${ }^{8-12}$ no report is seen about the graft polymers having siloxane segments of wellcontrolled length.

We already reported methods to synthesize dimethylsiloxane oligomers and macromonomers which can be polymerized not only by vinyl polymerization ${ }^{13}$ but also by polycondensation. ${ }^{14,15}$ In this article, we would like to report the effects of the introduction of oligodimethylsiloxane of defined length (1-4 $\mathrm{Si}$ atoms) into main-chain or side-chain of aromatic polyamide and polyimide on permeation behavior through the polymer membrane.
The structure of the polymers synthesized in this study is shown in Figure 1.

Structure of the diamino compound was selected to synthesize polymers having desired structure. In the figure, m-, s-, m, s-indicates polymers containing siloxane component in main-chain, side-chain, and main- and sidechains (polymer from the reaction using $1: 1$ mixture of 1,1-bis(4-aminophenyl)-substituted oligodimethylsiloxane and $\alpha, \omega$-bis(4-aminophenyl)-substituted oligodimethylsiloxane as diamino compounds), respectively. $M$ indicates polymers with oligosiloxane linked to mainchain through methine carbon. 


\section{EXPERIMENTAL}

\section{Synthesis}

Synthesis of aromatic diamino compounds: bis(4-aminophenyl)dimethylsilane, 1,3-bis(4aminophenyl)tetramethyldisiloxane, 1,5-bis(4aminophenyl)hexamethyltrisiloxane, 1,7bis(4-aminophenyl)octramethyltetrasiloxane; 1,1-bis(4-aminophenyl)tetramethyldisiloxane, 1,1-bis(4-aminophenyl)hexamethyltrisiloxane; and bis(4-aminophenyl)methylpentamethyldisiloxane, 1-[bis(4-aminophenyl)methyl]heptamethyltrisiloxane was already reported. ${ }^{14}$

Polymers were obtained by polycondensation reaction of the diamino compounds with terephthaloyl chloride (PTA series) or isophthaloyl chloride (PIA, PIAM series), or with pyromellitic dianhydride followed by thermal treatment at $200^{\circ} \mathrm{C}$ (PIM series) as typically shown in Schemes 1 and 2.

Typical procedures of the synthesis are given.

Poly(iminoisophthaloylimino-1,4-phenylene-

1,5-hexamethyltrisiloxanylene-1,4-phenylene) (m-PIA3)

After a flask containing 1,5-bis(4-aminophenyl)hexamethyltrisiloxane $(0.777 \mathrm{~g}, 1.989$ $\mathrm{mmol})$ and lithium chloride $(0.21 \mathrm{~g})$ was evacuated under vacuum, nitrogen gas was introduced. Triethylamine $(0.44 \mathrm{~g}, 4.4 \mathrm{mmol})$ and $N$-methyl-2-pyrrolidone $\left(8 \mathrm{~cm}^{3}\right)$ were added, and stirring was commenced. To the<smiles>CCNCCOC(C)(C)C(=O)C(=O)Cl</smiles><smiles>CC(C)N(C)c1ccc([Si](C)(C)O[Si](C)(C)O[Si](C)(C)C(C)(C)C2C=CC(NC(=O)C=CC(=O)[O-])C2)cc1</smiles>

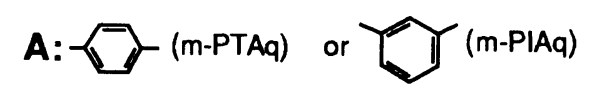

Scheme 1. Synthesis of polyamide containing oligodimethylsiloxane.<smiles>COC1C(=O)c2cc3c(cc2C1=O)C(=O)OC3=O</smiles>

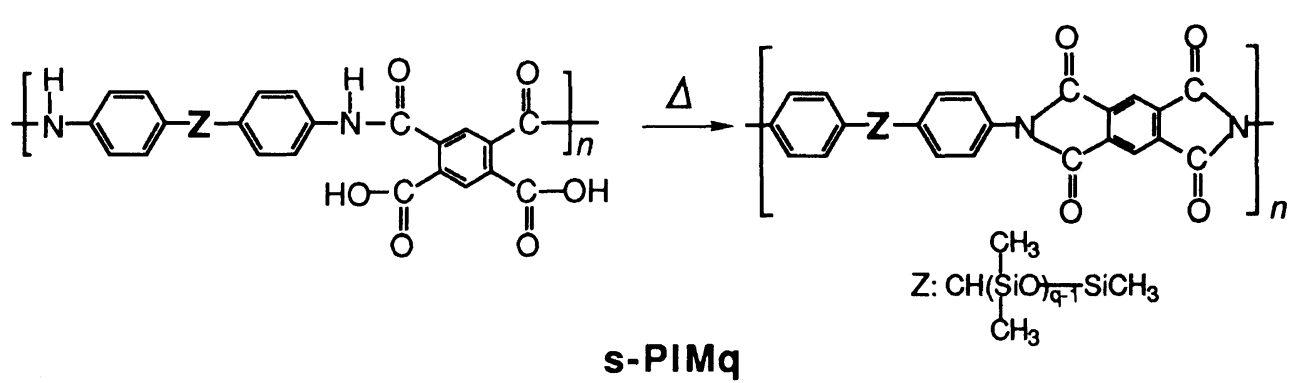

Scheme 2. Synthesis of polyimide containing oligodimethylsiloxane. 
system, isophthaloyl chloride $(0.404 \mathrm{~g}, 1.989$ mmol) was added during 1-2 min under ice-cooling. The reaction system became viscous about after $20 \mathrm{~min}$. It was allowed to warm-up to room temperature, and further reacted at the temperature for $3 \mathrm{~h}$. The produced polymer was recovered by pouring into methanol after the dilution of the system with $N$-methyl-2-pyrrolidone $\left(10 \mathrm{~cm}^{3}\right)$. The crude polymer was purified by reprecipitation procedure ( 3 times) from tetrahydrofuran into methanol. Yield, $0.80 \mathrm{~g} ; 77 \%$.

Equimolar mixture of diamino compounds were used to synthesize $\mathrm{m}$, s-type polymers.

\section{Poly(pyromelliticimido-1,4-phenylene-penta-} methyldisiloxanylmethylene-1,4-phenylene) (PIM2)

To pyromellitic dianhydride $(0.655 \mathrm{~g}, 3.00$ mmol) placed in an evacuated and nitrogen susbstituted flask, [bis(4-aminophenyl)methyl]pentamethyldisiloxane $(1.034 \mathrm{~g}, 3.00 \mathrm{mmol})$ in dimethylacetamide $\left(10 \mathrm{~cm}^{3}\right)$ was added, and the reaction system was gently stirred for $12 \mathrm{~h}$ at $60^{\circ} \mathrm{C}$. Fifteen $\%$ solution of polyamic acid was obtained by the concentration of the solution.

\section{Analysis and Measurement}

NMR spectra were recorded on a Varian $200 \mathrm{MHz}$ spectrometer (model Gemini 200) in dimethyl sulfoxide- $d_{6}$ (DMSO- $\left.d_{6}\right)$. Glass transition temperature $\left(T_{\mathrm{g}}\right)$ of polymers were estimated on a Seiko Instruments DSC (model DSC 100), at a heating rate $10 \mathrm{~K} \mathrm{~min}^{-1}$. Intrinsic viscosity was determined in dimethylacetamide at $30^{\circ} \mathrm{C}$. Gas permeation behavior was estimated for supplied air by measuring the amounts of gas permeated by gas chromatography, maintaining the upstream pressure at $1 \mathrm{~atm}$ and the downstream at zero, at $25^{\circ} \mathrm{C}$, on a Yanaco gas permeability measuring system (model GTR-20). The permeability, diffusion and solubility coefficients of oxygen in air were shown as $P_{\mathrm{O}_{2}}, D_{\mathrm{O}_{2}}$, and $S_{\mathrm{O}_{2}}$, respectively. The detailed method of the measurement and treatment of the data are reported in ref 3 and 7 .

\section{Preparation of Film}

Polyamide: A polymer (0.25 g) was dissolved in $3 \mathrm{~cm}^{3}$ dimethylacetamide (PIA0, PIA1, PTA1, m-PIA2, m-PTA2) or tetrahydrofuran (other polyamides), and the solution was spread on a clean glass surface. The solvent was allowed to evaporate at room temperature under weak stream of nitrogen gas in a desiccator. The formed film was removed from glass by immersing into water, dried under vacuum over night, and conditioned at a temperature a little below the glass transition temperature of the polymer for $10 \mathrm{~h}$.

Polyimide: The $15 \%$ solution of the polyamic acid in acetamide was spread on a clean glass maintained at $80^{\circ} \mathrm{C}$ in a oven. The temperature of the oven was elevated gradually to $240^{\circ} \mathrm{C}$ during $5 \mathrm{~h}$. After the film was heated at the temperature for further $2 \mathrm{~h}$ under vacuum, the film was removed from the glass.

\section{RESULTS AND DISCUSSION}

The results of the synthesis of polyamides are shown in Tables I and II.

It is well recognized that equimolarity between the acid and amine components is crucial to obtain high molecular weight polymer in polycondensation reaction. Since amines in this study are usually viscous oil or waxy material, caution was specially given to weigh the amine component. First, the amine was dissolved in dimethylacetamide, gently stirred with calcium hydride, and an aliquot amount of the supernatant solution was transferred to the reaction flask with a syringe having calibrated volume. The concentration of the amine solution, determined by titration, was made as high as possible.

The acid component was added all at once. Pyromellitic dianhydride was added as a lump. This seems essential to obtain high molecular weight polymer. Lithium chloride is also 
Table I. Synthesis of polyamides with oligodimethylsiloxane in main-chain

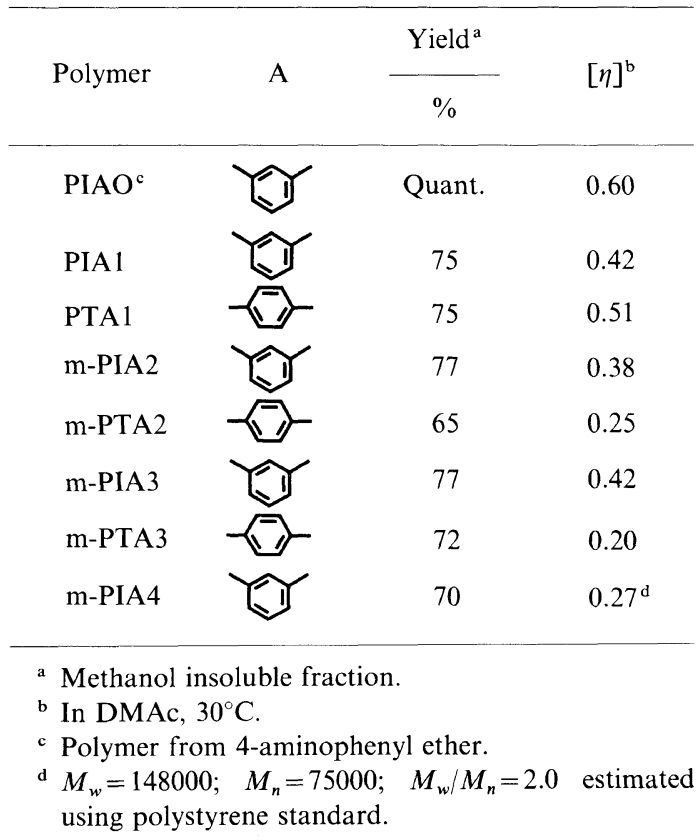

Table II. Synthesis of polyamides with oligodimethylsiloxane in side-chain(s-PIAq, s-PIAMq)

\begin{tabular}{lcc}
\hline \multicolumn{1}{c}{ Polymer } & ${\text { Yield } / \%^{\mathrm{a}}}^{\mathrm{a}}$ & {$[\eta]^{\mathrm{b}}$} \\
\hline PIA1 & 75 & 0.42 \\
s-PIA2 & 70 & 0.32 \\
s-PIA3 & 75 & 0.31 \\
s-PIAM1 & 74 & 0.64 \\
s-PIAM2 & 77 & 0.42 \\
s-PIAM3 & 72 & 0.37 \\
m, s-PIA22 & 73 & 0.51
\end{tabular}

a Methanol insoluble fraction.

${ }^{b}$ In DMAc, $30^{\circ} \mathrm{C}$.

essential component to obtain high molecular weight polymer. Reasonably high molecular weight polymers were obtained with these precautions. The general trend, that the intrinsic viscosity of the polymer of higher siloxane content is lower than that of the polymer of lower content, may reflect the intrinsic property of the polymer or the lower molecular weight of the polymer of higher
Table III. Oxygen permeability of polymers with oligodimethylsiloxane

\begin{tabular}{cccccc}
\hline Polymer & $T_{\mathrm{g}} /{ }^{\circ} \mathrm{C}$ & $P_{\mathrm{O}_{2}}$ & $D_{\mathrm{O}_{2}}$ & $S_{\mathrm{O}_{2}}$ & $P_{\mathrm{O}_{2}} / P_{\mathrm{N}_{2}}$ \\
\hline PIAO $^{a}$ & - & 0.15 & - & - & 5.8 \\
PIA1 & 234 & 0.46 & 3.4 & 1.3 & 4.8 \\
m-PIA2 & 199 & 1.8 & 6.1 & 3.0 & 4.3 \\
m-PIA3 & 149 & 6.6 & 21 & 3.1 & 4.2 \\
m-PIA4 & 115 & 15 & 41 & 3.7 & 3.6 \\
PTA1 & - & 0.11 & 1.1 & 1.0 & 6.5 \\
m-PTA2 & - & 1.5 & - & - & 4.8 \\
m-PTA3 & - & 5.0 & - & - & 3.9 \\
s-PIA2 & 166 & 5.5 & 18 & 3.0 & 4.2 \\
s-PIA3 & 104 & 11 & 37 & 2.9 & 3.6 \\
s-PIAM1 & - & 1.1 & 7.2 & 1.5 & 5.6 \\
s-PIAM2 & 130 & 1.9 & 12 & 1.6 & 4.3 \\
s-PIAM3 & - & 3.5 & 23 & 1.5 & 4.1 \\
m, s-PIA22 & 212 & 3.2 & 8.9 & 3.5 & 5.2 \\
PIMO & - & 0.39 & 2.2 & 1.8 & 7.9 \\
PIM1 & - & 3.3 & 9.0 & 3.7 & 4.1 \\
PIM2 & - & 7.8 & 23 & 3.4 & 4.0 \\
PIM3 & - & 12 & 39 & 3.0 & 3.8
\end{tabular}

a Polymer from 4-aminophenyl ether.

b Polymer from bis(4-aminophenyl)methane. $P_{\mathrm{O}_{2}}$, in $10^{-10} \mathrm{~cm}^{3}(\mathrm{STP}) \cdot \mathrm{cm} \mathrm{cm}^{-2} \cdot \mathrm{s}^{-1} \cdot(\mathrm{cmHg})^{-1} ; \mathrm{D}_{\mathrm{O}_{2}}$, in $10^{-8} \mathrm{~cm}^{2} \mathrm{~s}^{-1} ; S_{\mathrm{O}_{2}}$, in $10^{-3} \mathrm{~cm}^{3}$ (STP) $\mathrm{cm}^{-3} \mathrm{cmHg}$.

siloxane content.

The NMR spectra of the polymers were reasonably assigned. In the $\mathrm{m}$, s polymer, two amine components are distributed in the same concentration reflecting the equimolar feed concentration. Polyimide could not be characterized since it was insoluble in any solvent examined.

Glass transition temperature and oxygen permeability are summarized in Table III.

The glass transition temperature of the polymers dropped sharply by the introduction of oligodimethylsiloxane components in the polymer. However, this drop is not as sharp as in the case of vinyl polymers like polystyrene, where $100^{\circ} \mathrm{C}$ drop in glass transition temperature is observed by the introduction of the disiloxane linkage. ${ }^{6,7}$ Contrary to vinyl polymers whose glass transition temperature is mainly controlled by the slipping motion of the polymer segments, or by the disentanglement of chains in the solid state, hydrogen bonding 
and dipole-dipole interaction between polymer segments seem to play essential roles in determining the glass transition temperature of polycondensation polymers. The effect is larger for the oligosiloxanes introduced in side-chain than those in main-chain (m-PIA2, $199^{\circ} \mathrm{C}$; s-PIA2, $166^{\circ} \mathrm{C}$; m-PIA3, $149^{\circ} \mathrm{C}$; s-PIA3, $104^{\circ} \mathrm{C}$ ). Since one end of the oligodimethylsiloxane in side-chain type is not fixed, they have more freedom to move, hence they contribute more to the segmental motion of the polymers resulting in lower glass transition temperature of the side-chain type polymer. Polymer with disiloxanes introduced into both main- and side-chains (m, s-PIA2) showed only one single glass transition temperature. Two diamino components seems to be distributed randomly in the polymer. These disiloxane components did not lower the glass transition temperature markedly. The reason is not clear at present.

When PIA3 and PIAM2 are compared, we can discuss the role of $-\mathrm{CHSiO}-$ and $-\mathrm{SiO}-$ in giving flexibility to the polymer main chain. The higher glass transition temperature by the introduction of $-\mathrm{Si}-$ on $\mathrm{CH}-$ as $-\mathrm{CHSiO}-$ (PIAM2) can be understood as a result of the attachment of bulky group which restrict the segmental motion of the main chain segments. This fact clearly indicate that the mobility of oligosiloxane in side-chains is one of the principal factors to control the glass transition temperature of polymers.

The role of the substituents in permeation is also shown in Table III. Permeability coefficients became larger with the increase in the number of siloxane linkage in the polymer. In general, increase in the permeability coefficient was brought about by increase in the diffusion coefficient as in the case of oligosiloxane substituted polystyrene. ${ }^{6,7}$ For instance, the permeability coefficient of PIA1 (0.46) was increased to 1.8 (m-PIA2), 6.6 (m-PIA3), and 15 (m-PIA4) by the introduction of siloxane, disiloxane, and trisiloxane linkage (about 8 times increase). Meanwhile, the change in the solubility coefficient is at most three times in the value. Even in PTA series which would have high glass transition temperature and low permeability coefficient without siloxane linkage, the siloxane linkage is playing the main role in increasing the permeability coefficient by increasing the diffusion coefficient. When we compare the difference between PIA and PTA series, the difference became smaller by the increase in the length of the siloxane segments. These facts strongly suggest that the permeation is mainly controlled by the mobility of the siloxane linkages. Since, the permeability coefficient is principally controlled by the diffusion coefficient, the selectivity in permeation $\left(P_{\mathrm{O}_{2}} / P_{\mathrm{N}_{2}}\right)$ became lower with increase in the permeability coefficient. Evaluation of dynamic mobility of local segments by solid state NMR will make more detailed discussion possible. $^{7}$

When we compare the effects of siloxane linkage in main-chain and side-chain, increase in permeability and diffusion coefficients is higher when they are introduced into sidechain. This also reflects the higher mobility of oligosiloxane in side-chain than in main-chain.

Such principle can be applied to other polymers. ${ }^{5}$ Introduction of pentamethyldilsiloxanyl substituents into polyimide as sidechains through methine carbon increased the permeability about 10 times. Comparing with the large enhancement in the permeability of vinyl polymers by the introduction pentamethyldisiloxanyl substituents in side-chain, for example, 30 times increase was observed for polystyrene, the smaller increase in the permeability coefficient of polycondensation polymers indicates that hydrogen bonding and or dipole-dipole interaction between amide or imide groups seems to be playing another major role in controlling dynamic gaps between polymer chains (free volume in dynamic sense) in controlling the permeability of gas through the polymer membrane. When oligosiloxane is introduced into both main- and side-chains, the effects seem a little larger than the additive of the effects of the both substituents, which 
suggests that oligosiloxane in main-chain and side-chain are working in cooperative mode to decrease the glass transition temperature and to increase the permeability coefficient by weakening the intermolecular interactions.

These polymers are stable even at $300^{\circ} \mathrm{C}$ except m, s-PIA22 (stable up to $275^{\circ} \mathrm{C}$ ). This is a very important property of these polymers when an application is considered.

\section{CONCLUSION}

It was shown that the introduction of oligodimethylsiloxane of definite length and structure into polymer is a general way to increase and control the permeability of gases through the polymer membrane in maintaining the self-supporting film forming property of the polymer of high glass transition temperature.

Acknowledgments. Financial support from a Grant-in-Aid for Scientific Research on Priority Areas, New Functionality MaterialsDesign, Preparation and Control (04205065) is gratefully acknowledged.

\section{REFERENCES}

1. S. A. Stern, V. M. Shah, and B. J. Hardy, J. Polym. Sci., Polym. Chem. Ed., 25, 1263 (1987).

2. Y. Kawakami, H. Karasawa, H. Kamiya, T. Aoki, Y. Yamamura, and Y. Yamashita, Polym. Commun., 26, 133 (1985).

3. Y. Kawakami, H. Karasawa, T. Aoki, Y. Yamamura, H. Hisada, and Y. Yamashita, Polym. J., 17, 1159 (1985).

4. Y. Kawakami, H. Karasawa, H. Kamiya, T. Aoki, and Y. Yamashita, Polym. J., 18, 237 (1986).

5. Y. Kawakami, H. Toda, M. Higashino, and Y. Yamashita, Polym. J., 20, 285 (1988).

6. Y. Kawakami, T. Sugisaka, and Y. Yamashita, Polym. J., 20, 685 (1988).

7. Y. Kawakami and T. Sugisaka, J. Membrane Sci., 50, 189 (1990)

8. W. J. Ward, III, W. R. Browall, and R. M. Salemme, J. Membrane Sci., 1, 99 (1976).

9. M. Kajiyama, Y. Nishikata, M. Kakimoto, and Y. Imai, Polym. J., 18, 735 (1986).

10. M. Kakimoto, M. Kajiyama, and Y. Imai, Polym. J., 18, 935 (1986).

11. J. L. Hedrick, B. Haider, T. P. Russell, and D. C. Hofer, Macromolecules, 21, 1967 (1988).

12. Y. Nagase and K. Matsui, Polym. Prepr. Jpn., 36, 3357 (1987).

13. Y. Kawakami, Y. Miki, T. Tsuda, R. A. N. Murthy, and Y. Yamashita, Polym. J., 14, 913 (1983).

14. Y. Kawakami, R. A. N. Murthy, and Y. Yamashita, Makromol. Chem., 185, 9 (1984).

15. Y. Kawakami, S.-P. Yu, and T. Abe, Polym. Bull., 1992, submitted. 